\title{
325 - Relationship quality in dementia: Preliminary longitudinal analyses of the EU-JPND Actifcare cohort study
}

\author{
AUTHORS \\ Maria J. Marques \\ Bob Woods \\ Eva Y.L. Tan \\ Marjolein de Vugt \\ Frans Verhey \\ Manuel Gonçalves-Pereira \\ Actifcare Group \\ INTRODUCTION \\ Relationship quality $(\mathrm{RQ})$ in dyads of persons with dementia and their family carers is important both as \\ a clinical outcome and as a determinant of health and quality of life. In previous work we studied RQ \\ using baseline data of a large-scale European longitudinal study on timely access to and use of \\ community formal services in dementia (EU-JPND Acticare). We concluded that neuropsychiatric \\ symptoms and carer stress contributed to discrepancies in RQ ratings within the dyad, which were less \\ favourable when reported by family carers. This and other associations (e.g. between carer-rated RQ \\ and sense of coherence) were cautiously interpreted, in the context of a cross-sectional analysis.
}

\section{OBJECTIVES}

To analyse how carer-reported $R Q$ varies over time and to examine its most important influencing factors.

\section{METHODS}

We present preliminary longitudinal analyses from the Actifcare cohort study of 451 communitydwelling persons with dementia and their primary carers in eight European countries (12-month followup). Comprehensive assessments included the Positive Affect Index (PAI) to assess RQ, persons with dementia's neuropsychiatric symptoms, persons with dementia and carers' unmet needs, carers' anxiety and depression, social support, sense of coherence and stress.

\section{RESULTS}

Carers' mean PAI scores decreased over the 12-month period. The person with dementia neuropsychiatric symptoms and unmet needs, and carers' perceived social support were significant predictors of carers' RQ change.

\section{DISCUSSION AND CONCLUSION}

We analysed carer-reported $R Q$ variation over time and predictors in a large European sample of persons with dementia and their family carers. As expected, RQ decreased over the oneyear follow-up period as the disease progressed. Its main predictors in this sample (neuropsychiatric symptoms and the person's unmet needs, together with carers' social support) can all influence the impact that caregiving has on the carer and on how time and energy-consuming caregiving is. The role of increased clinical symptoms (also affecting communication difficulties), together with carers' exhaustion, must be equated. Overall, these results may help us to tailor interventions addressing $R Q$ and potentially improve dementia outcomes. 